\section{Fatores associados ao tabagismo em idosos: Inquérito de Saúde no Estado de São Paulo (ISA-SP)}

\author{
Factors associated with smoking in the elderly: \\ a health survey in São Paulo (ISA-SP)
}

\footnotetext{
1 Faculdade de Ciências Médicas, Universidade Estadual de Campinas, Campinas, Brasil.

2 Faculdade de Saúde Pública, Universidade de São Paulo, São Paulo, Brasil. 3 Faculdade de Medicina de Botucatu, Universidade Estadual Paulista Júlio de Mesquita Filho, Botucatu, Brasil.

4 Faculdade de Medicina, Universidade de São Paulo, São Paulo, Brasil. 5 Instituto de Saúde, Secretaria de Estado da Saúde de São Paulo, São Paulo, Brasil.

Correspondência M. P. A. Zaitune Departamento de Medicina Preventiva e Social, Faculdade de Ciências Médicas, Universidade Estadual de Campinas. SHIS QI 25, conjunto 13, casa 9, Brasília, DF 71660-330, Brasil. mpzaitune@gmail.com
}

\begin{abstract}
This article reports on smoking prevalence and associated factors in the elderly, based on a population-based cross-sectional study with multistage sampling including 1,954 individuals 60 years or older living in four areas of São Paulo State, Brazil. Overall smoking prevalence was $12.2 \%$, and higher rates were associated with male gender, age 60-69 years, not belonging to an Evangelical church, lower income, low body weight, lack of leisure-time physical activity, depression/anxiety, and hypertension. There was a high prevalence of smokers among individuals with a history of stroke, cancer, and chronic obstructive pulmonary disease. The results point to the need for effective interventions in healthcare services to promote smoking cessation among the elderly, since many are unable to stop on their own, even when they have tobacco-related illnesses. Special attention should be paid to individuals that depend on the National Health System, since smoking prevalence is higher in underprivileged socioeconomic groups.
\end{abstract}

Aged; Smoking; Morbidity Surveys; Health Promotion
Maria Paula do Amaral Zaitune 1

Marilisa Berti de Azevedo Barros 1

Margareth Guimarães Lima 1

Chester Luiz Galvão César 2

Luana Carandina 3

Moisés Goldbaum 4

Maria Cecilia Goi Porto Alves 5

\section{Introdução}

O tabagismo é o mais importante fator de risco para 7 das 14 principais causas de morte entre os idosos 1 , constituindo um dos principais problemas de saúde pública da atualidade 2 .

Calcula-se que ocorrerão aproximadamente 7 milhões de óbitos atribuíveis ao consumo de tabaco entre 2020 e 2030 nos países em desenvolvimento ${ }^{3}$. No Brasil, o tabagismo constitui uma das principais causas de mortes evitáveis, estimando-se que seja responsável por $45 \%$ dos óbitos por infarto do miocárdio, $85 \%$ dos provocados por enfisema, $25 \%$ das mortes por doença cerebrovascular e $30 \%$ das provocadas por câncer 4 .

Na Classificação Internacional de Doenças (10a revisão - CID-10), o tabagismo situa-se no grupo dos transtornos mentais e de comportamentos relacionados ao uso de substâncias psicoativas. Além de causar dependência à nicotina ${ }^{5}$, o tabaco expõe o usuário a cerca de 4.700 substâncias tóxicas, sendo 60 destas carcinogênicas. A exposição ao tabaco predispõe assim à ocorrência de doenças limitantes e fatais, como doenças respiratórias crônicas, vasculares periféricas, cerebrovasculares, cardiopatias e neoplasias, entre outras $6,7,8,9$.

A maior parte dos estudos sobre tabagismo conduzidos no Brasil e no exterior prioriza jovens e adultos como população pesquisada, e pouco destaque tem sido dado quanto a este tema en- 
tre idosos 10 . Idosos tabagistas, por terem sofrido em suas vidas exposições mais longas ao fumo, a cigarros sem filtro e com elevados teores de nicotina têm maior risco atual de apresentar doenças relacionadas ao cigarro 11,12 do que coortes de gerações posteriores. $\mathrm{O}$ consumo de tabaco pelos idosos tende a favorecer o surgimento de comorbidades, ampliando os gastos deste grupo etário com cuidados de saúde 12 .

Os idosos ainda fumantes, sendo constituídos por aqueles que conseguiram sobreviver às taxas excessivas das mortes prematuras provocadas pelo tabaco, tendem a ser pouco motivados a parar de fumar, subestimando os próprios riscos e considerando-se relativamente imunes aos prejuízos causados pelo cigarro ${ }^{12}$. Os profissionais de saúde, por sua vez, tendem a não se empenhar na cessação do tabagismo ao considerar a idade "avançada" do paciente e a não querer privá-lo de uma fonte de prazer.

Mas, trata-se de faixa etária que já apresenta maior prevalência de agravos crônicos, não necessariamente relacionados ao tabaco, e a continuidade do uso do cigarro contribui para o maior risco de complicações, de surgimento de comorbidades e dos prejuízos terapêuticos decorrentes do efeito do fumo no metabolismo de vários medicamentos ${ }^{1}$. Além disso, o tabagismo tende a ocorrer na concomitância de vários outros comportamentos não saudáveis como inatividade física, ingestão insuficiente de frutas e hortaliças, consumo excessivo de bebidas alcoólicas e de gorduras saturadas de origem animal $13,14,15$, elevando ainda mais a probabilidade do indivíduo desenvolver doenças crônicas.

Embora a prevalência do comportamento de fumar nos idosos seja menor do que nos adultos em decorrência de óbito precoce dos fumantes, da cessação do consumo de cigarros com o surgimento de doenças, da opção de aderir a comportamentos mais saudáveis e do efeito coorte, o número absoluto de idosos fumantes tende a aumentar com o envelhecimento da população 1,16,17. No Brasil, o crescimento do número de idosos ocorreu de forma acelerada com um aumento expressivo de aproximadamente $700 \%$ em menos de meio século (3 milhões em 1960 para 20 milhões em 2008) e as projeções apontam que este contingente superará 30 milhões de idosos em 202018.

Por outro lado, pesquisas têm demonstrado benefícios com a cessação do uso de cigarros em todas as idades, inclusive em pessoas já apresentando doenças relacionadas ao tabaco 1,12. Diretrizes para a cessação de tabagismo 19 assinalam que os prejuízos causados pelo fumo podem ser minimizados mesmo naqueles que fumaram três décadas ou mais, nos quais a cessação pode di- minuir os riscos de desenvolvimento de novas comorbidades e melhorar o prognóstico das doenças já existentes, promovendo melhora da qualidade de vida dos idosos.

Considerando as implicações do tabagismo no idoso e os benefícios que a cessação poderia trazer, o presente estudo teve por objetivo determinar a prevalência do tabagismo e os fatores associados nesse grupo etário, com o intuito de identificar segmentos demográficos mais vulneráveis e subsidiar o desenvolvimento de estratégias de cessação do tabagismo e de promoção da saúde.

\section{Métodos}

Trata-se de um estudo transversal, de base populacional, que incluiu indivíduos de 60 anos e mais, não institucionalizados, residentes em quatro áreas do Estado de São Paulo: (1) região sudoeste da Grande São Paulo, constituída pelos municípios de Taboão da Serra, Itapecerica da Serra e Embu; (2) Administração Regional do Butantã no Município de São Paulo, composta pelos distritos do Butantã, Jaguaré, Rio Pequeno, Raposo Tavares, Vila Sônia e Morumbi; (3) Município de Campinas; e (4) Município de Botucatu. Esta pesquisa é parte de um estudo multicêntrico - Inquérito de Saúde no Estado de São Paulo (ISASP), realizado em 2001-2002 20.

Para este inquérito foi estimado um tamanho mínimo de amostra de 196 pessoas para cada domínio de idade e sexo, para cada uma das quatro áreas estudadas. Como o trabalho tinha por objetivo estimar prevalências de diferentes morbidades e comportamentos de saúde, o tamanho da amostra foi calculado de forma conservadora considerando a estimação de uma prevalência de $50 \%$ (que corresponde à máxima variabilidade) com erro máximo de 0,10 , nível de $95 \%$ de confiança e efeito de delineamento de dois. Prevendo uma possível perda de $20 \%$, foram sorteados 250 indivíduos de cada domínio. Os domínios de amostragem foram: menor de 1 ano, 2 a 11 anos, 12 a 19 anos masculino, 12 a 19 anos feminino, 20 a 59 anos masculino, 20 a 59 anos feminino, 60 anos e mais masculino e 60 anos e mais feminino. Para o presente estudo foram incluídos apenas os domínios de 60 anos e mais, de ambos os sexos.

A amostragem foi probabilística, por conglomerados, estratificada e em dois estágios: setores censitários e domicílios. Os setores censitários foram classificados e agrupados em três estratos segundo o percentual de chefes de família com nível universitário: menos de 5\%, de 5\% a 25\% e mais de $25 \%$. De cada estrato foram sorteados dez setores censitários. Após atualização, em campo, 
dos mapas dos setores sorteados e elaboração da listagem de endereços, foram selecionados os domicílios que compuseram a amostra. No domicílio, foram entrevistados todos os moradores pertencentes ao(s) domínio(s) sorteado(s) para aquele domicílio. Detalhes do procedimento amostral encontram-se em outra publicação 21.

As informações foram obtidas por meio de questionário aplicado por entrevistadores treinados diretamente ao indivíduo selecionado pelo processo amostral. No caso de idosos impossibilitados de responder às questões, a entrevista foi realizada com o familiar ou o cuidador responsável. O questionário foi composto principalmente por questões fechadas e organizadas em blocos temáticos que incluíram características socioeconômicas, morbidade, estilo de vida e uso de serviços de saúde.

As variáveis incluídas no presente estudo foram:

a) Condição tabágica: fumante, ex-fumante e nunca fumou. Foi considerada fumante ou exfumante aquela pessoa que informou que havia fumado pelo menos um cigarro por dia durante pelo menos um mês, sendo considerado fumante atual a que persistia no comportamento por ocasião da entrevista. Também foram analisadas as variáveis: início do comportamento de fumar, tempo que leva para acender o primeiro cigarro após acordar e número de cigarros consumidos por dia.

b) Socioeconômicas e demográficas: sexo, idade, cor/raça autorreferida, situação conjugal, religião, escolaridade, renda familiar mensal per capita (em salários mínimos) e atividade ocupacional.

c) Comportamentos relacionados à saúde: (1) índice de qualidade da dieta (IQD) obtido por pontuação em dez componentes totalizando até 100 pontos. O escore total foi categorizado em: dieta "inadequada" (abaixo de 51 pontos), dieta que "necessita de modificação" (entre 51 a 80 pontos) e dieta "saudável" (superior a 80 pontos) 22 ; (2) frequência semanal de ingestão de bebida alcoólica e teste CAGE 23 considerado positivo com duas ou mais respostas afirmativas; (3) atividade física de lazer definida como a prática regular de qualquer esporte ou exercício físico pelo menos uma vez por semana; (4) nível de atividade física, avaliado pelo Questionário Internacional de Atividade Física - QIAF 24, utilizando-se a versão simplificada, composta por sete questões, que provê informações da frequência e duração de caminhadas e de atividades de intensidades moderada e vigorosa realizadas em uma semana habitual, mas sem especificar o contexto em que as atividades são realizadas. $\mathrm{O}$ instrumento permite classificar o indivíduo em sedentário, insu- ficientemente ativo, ativo e muito ativo. Para este estudo, considerou-se o agrupamento "ativos + muito ativos" e "sedentários + insuficientemente ativos".

d) Morbidades: (1) presença de doença relacionada no checklist - hipertensão arterial, diabetes melittus, acidente vascular encefálico (AVE), depressão/ansiedade, osteoporose, doença crônica do pulmão, câncer e doença do coração; número de doenças crônicas referidas; (2) transtorno mental comum (TMC) avaliado com base no Self Reporting Questionnaire (SRQ-20), com ponto de corte $7 / 825$.

e) Índice de massa corporal $\left(\mathrm{IMC}=\mathrm{kg} / \mathrm{m}^{2}\right)$ : calculado com dados de peso e altura referidos, sendo utilizadas as categorias de IMC preconizadas para o idoso com os seguintes pontos de corte: baixo peso $\left(<22 \mathrm{~kg} / \mathrm{m}^{2}\right)$, eutrofia ( $22 \leq$ IMC $\leq 27 \mathrm{~kg} / \mathrm{m}^{2}$ ) e sobrepeso $\left(>27 \mathrm{~kg} / \mathrm{m}^{2}\right) 26$.

Foram feitas estimativas de prevalências e testadas as associações entre as diversas variáveis e o tabagismo usando-se o teste qui-quadrado $\left(\chi^{2}\right)$ com nível de significância de 5\%; foram calculadas as razões de prevalência brutas e ajustadas para idade e sexo e respectivos intervalos de 95\% de confiança (IC95\%), usando-se regressão simples e múltipla de Poisson que permite estimativa mais conservadora e interpretável 27 . Apenas as variáveis que apresentaram o valor de $\mathrm{p}<$ 0,20 na análise bivariada foram incluídas no modelo de regressão múltipla, sendo mantidas no modelo aquelas que persistiram associadas com a variável dependente com valor de $\mathrm{p}$ menor que 0,05 . Todas as análises foram feitas com o software Stata 9.0 (Stata Corp., College Station, Estados Unidos), utilizando os comandos svy que permitem a incorporação dos fatores de ponderação da amostra, considerando além dos pesos, também os estratos e os conglomerados. Os pesos finais incorporaram ainda ajustes de pós-estratificação que consideraram as informações do Censo Demográfico de 2000 (Instituto Brasileiro de Geografia e Estatística. http:/ / www.ibge.gov.br) sobre a estrutura da população por idade e sexo.

O projeto foi aprovado pelo Comitê de Ética em Pesquisa da Faculdade de Ciências Médicas da Universidade Estadual de Campinas (FCMUNICAMP) sob o parecer $n^{\circ} .369 / 2000$.

\section{Resultados}

Dos idosos identificados nos domicílios sorteados, 9,1\% recusaram-se a participar do estudo e $0,3 \%$ não pôde ser entrevistado devido a não concessão da entrevista após mais de três tentativas. No total foram entrevistados 1.954 indivíduos com idade igual ou superior a 60 anos, dos quais 
1.027 eram do sexo feminino. A média de idade foi de 69,7 anos com desvio-padrão de 7,5 anos.

Na ocasião da entrevista, a população de idosos correspondia a 19.358 na Grande São Paulo, 32.474 no Butantã, 10.206 em Botucatu e 76.762 em Campinas, totalizando 138.800 idosos. Entre esses, o número de fumantes foi de 16.933 (14.018-20.403).

A Figura 1 mostra a prevalência da situação tabágica segundo sexo. A prevalência encontrada de fumantes foi de 12,2\% (IC95\%: 10,1-14,7), sendo maior no sexo masculino (17,5\%). Também foi encontrada maior prevalência de ex-fumantes em homens $(47,6 \%)$, sendo que $73,7 \%$ das mulheres nunca haviam fumado.

Dos idosos fumantes, $42,7 \%$ tinham iniciado o comportamento antes dos 16 anos de idade e $30,6 \%$ entre os 16 e 20 anos. Em relação à quantidade de cigarros consumidos, $42,5 \%$ dos fumantes atuais referiram fumar 20 ou mais cigarros diariamente e $36,8 \%$ de 5 a 19 cigarros/dia, sendo que $71 \%$ dos fumantes declararam já ter tentado parar de fumar alguma vez na vida (dados não mostrados em tabela).

Em relação às variáveis socioeconômicas e demográficas (Tabela 1), as prevalências de fumantes foram significativamente mais elevadas entre: idosos do sexo masculino, com 60 a 69 anos, não brancos, que tinham renda menor ou igual a um salário mínimo e que ainda estavam em atividade ocupacional. Essa última associação deixa de ser significante após ajuste por idade e sexo. Foi menos elevada nos evangélicos quando comparados aos católicos e aos de outras ou sem religião.

O tabagismo mostrou-se também significativamente mais frequente entre os idosos que bebiam de 4 a 7 vezes por semana, que tinham dieta inadequada, que não praticavam atividade física de lazer e que estavam na condição de baixo peso. Nos que apresentaram teste CAGE positivo a razão de prevalência foi maior apenas na análise bruta e perdendo a significância após ajuste por idade e sexo. A prevalência de fumantes nos idosos com sobrepeso foi significativamente menor que nos de peso normal (Tabela 2).

A Tabela 3 mostra a prevalência de fumantes segundo a presença de morbidades. Em relação a algumas morbidades, os portadores da doença apresentaram menor prevalência de fumantes do que os não portadores, como observado com a hipertensão e com a osteoporose (nesta última, a significância estatística da associação desaparece após o ajuste por idade e sexo). Para outras morbidades, no entanto, o portador da doença apresentou maior frequência de tabagismo que os não portadores, como verificado com a depressão/ansiedade, a doença crônica do pulmão, o transtorno mental comum e o acidente vascular encefálico.

Figura 1

Prevalência de tabagismo segundo sexo, em pessoas com 60 anos ou mais. Inquérito de Saúde no Estado de São Paulo (ISA-SP), 2001-2002.

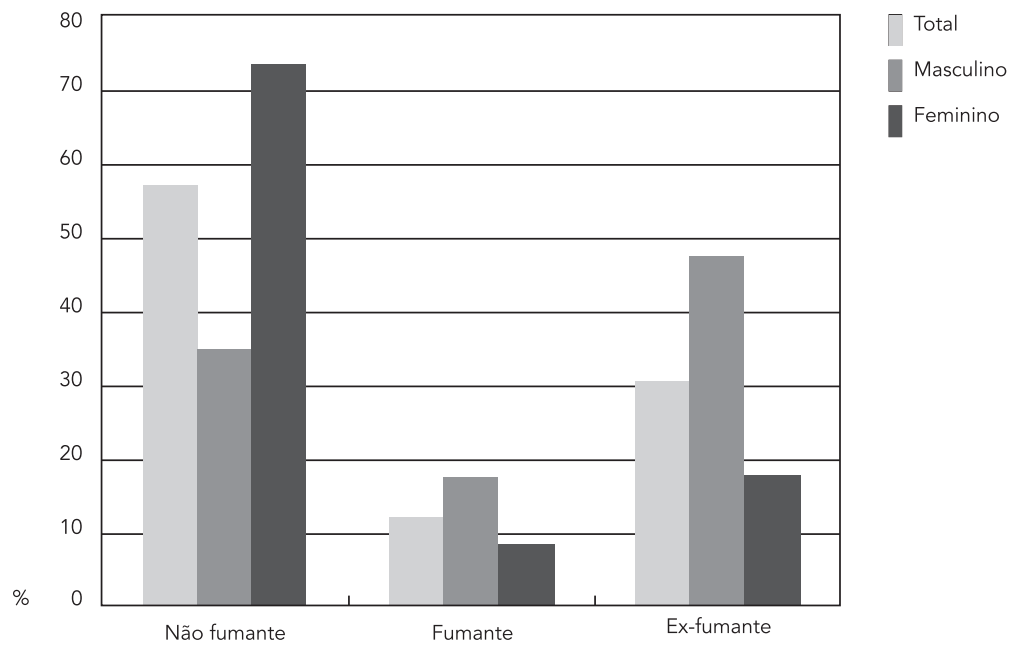


Prevalências de fumante e razões de prevalência (RP) segundo variáveis socioeconômicas e demográficas, em pessoas com 60 anos ou mais. Inquérito de Saúde no Estado de São Paulo (ISA-SP), 2001-2002.

\begin{tabular}{|c|c|c|c|c|}
\hline Variáveis e categorias & $n$ * & $\begin{array}{c}\text { Fumante } \\
\text { Prevalência (\%) }\end{array}$ & RP bruta (IC95\%) & $\begin{array}{l}\text { RP ajustada ** } \\
\text { (IC95\%) }\end{array}$ \\
\hline Sexo & $0,000 * \star \star$ & & & \\
\hline Masculino & 927 & $17,5(13,9-21,7)$ & 1,00 & 1,00 \\
\hline Feminino & 1.027 & $8,3(6,1-11,3)$ & $0,48(0,33-0,68)$ & $0,49(0,34-0,72)$ \\
\hline Total & 1.954 & $12,2(10,1-14,7)$ & & \\
\hline Faixa etária (anos) & $0,001 * \star \star *$ & & & \\
\hline $60-69$ & 1.091 & $15,3(12,2-19,0)$ & 1,00 & 1,00 \\
\hline $70-79$ & 643 & $9,1(6,7-12,3)$ & $0,59(0,40-0,89)$ & $0,62(0,41-0,94)$ \\
\hline 80 e mais & 220 & $6,0(3,0-11,6)$ & $0,39(0,21-0,72)$ & $0,42(0,34-0,72)$ \\
\hline Cor/Raça & $0,014 * \star \star$ & & & \\
\hline Branca & 1.508 & $11,1(8,9-13,8)$ & 1,00 & 1,00 \\
\hline Outras & 439 & $16,8(12,8-21,7)$ & $1,51(1,09-2,09)$ & $1,40(1,01-1,93)$ \\
\hline Situação conjugal & $0,304^{* \star *}$ & & & \\
\hline Com cônjuge & 1.170 & $13,1(10,4-16,4)$ & 1,00 & 1,00 \\
\hline Sem cônjuge & 773 & $11,1(8,4-14,4)$ & $0,84(0,60-1,17)$ & $1,35(0,94-1,95)$ \\
\hline Religião & $0,001 * \star \star$ & & & \\
\hline Católica & 1.426 & $13,8(11,1-17,1)$ & 1,00 & 1,00 \\
\hline Evangélica & 305 & $3,2(1,3-7,3)$ & $0,23(0,09-0,57)$ & $0,22(0,09-0,56)$ \\
\hline Outras/Sem religião & 201 & $13,0(8,2-20,0)$ & $0,93(0,58-1,50)$ & $0,91(0,57-1,45)$ \\
\hline \multicolumn{5}{|l|}{ Escolaridade do indivíduo (anos) } \\
\hline $0-3$ & 843 & $13,6(10,7-89,2)$ & 1,00 & 1,00 \\
\hline $4-7$ & 652 & $11,3(08,3-15,4)$ & $0,83(0,57-1,20)$ & $0,78(0,55-1,12)$ \\
\hline $8-11$ & 313 & $11,6(07,0-18,6)$ & $0,84(0,49-1,46)$ & $0,75(0,44-1,28)$ \\
\hline 12 ou mais & 138 & $10,3(05,3-18,9)$ & $0,75(0,38-1,48)$ & $0,60(0,29-1,23)$ \\
\hline Renda per capita (salários mínimos) & $0,000 * \star \star$ & & & \\
\hline$\leq 1$ & 566 & $18,5(14,3-23,6)$ & 1,00 & 1,00 \\
\hline$>1-2,5$ & 621 & $11,8(9,0-15,4)$ & $0,63(0,45-0,90)$ & $0,63(0,46-0,87)$ \\
\hline$>2,5-4$ & 303 & $11,3(7,2-17,2)$ & $0,61(0,37-0,98)$ & $0,63(0,38-1,02)$ \\
\hline$>4$ & 464 & $6,7(4,4-10,1)$ & $0,36(0,23-0,57)$ & $0,35(0,23-0,55)$ \\
\hline Em atividade ocupacional & $0,021 * \star \star$ & & & \\
\hline Não & 1.452 & $10,9(8,7-13,7)$ & 1,00 & 1,00 \\
\hline Sim & 502 & $16,3(12,3-21,4)$ & $1,49(1,06-2,08)$ & $0,99(0,69-1,41)$ \\
\hline
\end{tabular}

* Número de indivíduos na amostra não ponderada;

** Ajustes por sexo e idade;

$\star \star \star$ Valor de $\mathrm{p}$.

O modelo de regressão múltipla de Poisson (Tabela 4) permitiu observar prevalências de fumantes significativamente maiores nas categorias de idosos: do sexo masculino, de 60 a 69 anos, não evangélicos, de estratos inferiores de renda, com baixo peso corporal, que não praticavam atividade física de lazer, com depressão/ ansiedade e que referiram não ser hipertensos. Menores prevalências foram apresentadas pelos idosos com sobrepeso.

\section{Discussão}

A prevalência de tabagismo em idosos verificada neste estudo (12\%) foi semelhante à observada em Porto Alegre, Rio Grande do Sul (11,6\%) 28; na Região Metropolitana de Belo Horizonte, Minas Gerais (12,8\%) 29; na Pesquisa Especial de Tabagismo (PeTab, 12,9\% em maiores de 64 anos de idade) 30 ; e em inquérito que envolveu 16 capitais brasileiras $(12,7 \%)^{31}$, mas mostrouse inferior à encontrada na Pesquisa Mundial 
Prevalências de fumante e razões de prevalência (RP) segundo outros comportamentos de saúde, em pessoas com 60 anos ou mais. Inquérito de Saúde no Estado de São Paulo (ISA-SP), 2001-2002.

\begin{tabular}{|c|c|c|c|c|}
\hline Variáveis e categorias & $n$ * & $\begin{array}{c}\text { Fumante } \\
\text { Prevalência (\%) }\end{array}$ & RP bruta (IC95\%) & $\begin{array}{l}\text { RP ajustada ** } \\
\text { (IC95\%) }\end{array}$ \\
\hline $\begin{array}{l}\text { Frequência de ingestão de bebida } \\
\text { alcoólica (vezes/semana) }\end{array}$ & 0,004 *** & & & \\
\hline Não bebe & 1.217 & $9,9(7,4-13,0)$ & 1,00 & 1,00 \\
\hline Menos de 1 & 243 & $14,1(9,9-19,9)$ & $1,43(0,94-2,17)$ & $1,59(0,99-2,57)$ \\
\hline $1-3$ & 280 & $15,1(10,4-21,5)$ & $1,53(0,98-2,38)$ & $1,23(0,78-1,95)$ \\
\hline $4-7$ & 186 & $22,1(14,7-31,8)$ & $2,23(1,35-3,67)$ & $1,32(0,84-2,08)$ \\
\hline Teste CAGE & $0,003 * \star \star$ & & & \\
\hline Negativo & 1.852 & $11,7(9,6-14,1)$ & 1,00 & 1,00 \\
\hline Positivo & 66 & $27,5(16,0-42,9)$ & $2,34(1,39-3,96)$ & $1,67(0,98-2,85)$ \\
\hline Índice de qualidade da dieta & $0,0001 * \star \star$ & & & \\
\hline Dieta inadequada & 420 & $19,4(14,2-25,9)$ & 1,00 & 1,00 \\
\hline $\begin{array}{l}\text { Dieta saudável ou que necessita } \\
\text { de modificação }\end{array}$ & 1.534 & $10,2(0,8-12,47)$ & $0,52(0,38-0,71)$ & $0,50(0,30-0,70)$ \\
\hline Nível de atividade física (QIAF) & $0,221 * \star \star$ & & & \\
\hline Muito ativo/Ativo & 1.467 & $11,6(9,5-14,2)$ & 1,00 & 1,00 \\
\hline Insuficientemente ativo/Sedentário & 481 & $14,1(10,4-18,8)$ & $1,20(0,89-1,63)$ & $1,17(0,88-1,56)$ \\
\hline Atividade física de lazer & $0,000 * \star \star$ & & & \\
\hline Não pratica & 1.345 & $14,8(12,2-17,8)$ & 1,00 & 1,00 \\
\hline Pratica & 603 & $5,9(4,0-8,8)$ & $0,40(0,26-0,60)$ & $0,36(0,24-0,55)$ \\
\hline IMC & $0,000 * \star \star$ & & & \\
\hline Normal $\left(22 \leq \mathrm{IMC} \leq 27 \mathrm{~kg} / \mathrm{m}^{2}\right)$ & 787 & $11,4(8,8-14,6)$ & 1,00 & 1,00 \\
\hline Baixo peso $\left(<22 \mathrm{~kg} / \mathrm{m}^{2}\right)$ & 294 & $28,7(22,1-36,3)$ & $2,53(1,84-3,47)$ & $2,61(1,90-3,59)$ \\
\hline Sobrepeso $\left(>27 \mathrm{~kg} / \mathrm{m}^{2}\right)$ & 577 & $5,3(3,3-8,5)$ & $0,47(0,28-0,78)$ & $0,46(0,27-0,78)$ \\
\hline
\end{tabular}

IMC: índice de massa corporal; QIAF: Questionário Internacional de Atividade Física.

* Número de indivíduos na amostra não ponderada;

** Ajustes por sexo e idade;

$\star \star *$ Valor de $\mathrm{p}$.

de Saúde (15,4\%) 32, em Bambuí, Minas Gerais (18,7\%) 16; em Botucatu, São Paulo (16,4\%) 33; e no Estado do Rio Grande do Sul (18,8\%) 34, e superior à verificada nos Estados Unidos (8,3\%) 35 e para o Inquérito Telefônico de Vigilância de Fatores de Risco e Proteção para Doenças Crônicas (VIGITEL, 8,1\%) 36 com pessoas com 65 anos e mais.

Os resultados deste trabalho apontam para diferenças entre os gêneros com prevalências maiores no sexo masculino, assim como evidenciado em outros inquéritos 11,16,28,34,37,38,39. Entretanto, pesquisa realizada nos Estados Unidos não verificou diferença estatisticamente significante entre as prevalências de homens (9,3\%) e mulheres $(7,6 \%)$ de 65 anos e mais 35 . A maior proporção de homens fumantes que a de mulheres é explicada por fatores históricos e socioculturais ${ }^{31}$. O tabagismo difundiu-se primeira- mente entre os homens na segunda década do século XX e tornou-se mais comum tanto para homens como para mulheres após a Segunda Guerra Mundial 38, resultando em prevalências diferentes nas diversas coortes de nascimento.

Encontraram-se prevalências decrescentes de fumantes com o aumento da idade, assim como registrado na literatura 11,16,33,35,38,39,40, sendo aventados como fatores contributivos para estes resultados: o surgimento de agravos que levam à recomendação de cessação do consumo de cigarros, a maior probabilidade de óbito precoce dos fumantes 29,31,41, a atual e crescente preocupação com a saúde levando a adotar comportamentos mais saudáveis, e o efeito de coorte visto que em gerações mais antigas o tabagismo havia sido menos prevalente que após a Segunda Guerra Mundial 29. 
Prevalências de fumante e razões de prevalência (RP) brutas e ajustadas, segundo a presença de morbidades referidas, em pessoas com 60 anos ou mais. Inquérito de Saúde no Estado de de São Paulo (ISA-SP), 2001-2002.

\begin{tabular}{|c|c|c|c|c|}
\hline Variáveis e categorias & $n$ * & $\begin{array}{c}\text { Fumante } \\
\text { Prevalência (\%) }\end{array}$ & RP bruta (IC95\%) & $\begin{array}{l}\text { RP ajustada ** } \\
\text { (IC95\%) }\end{array}$ \\
\hline Número de doenças crônicas & $0,063 * \star \star$ & & & \\
\hline Nenhuma & 760 & $16,5(12,4-21,6)$ & 1,00 & 1,00 \\
\hline $1-2$ & 696 & $12,2(8,9-16,6)$ & $0,74(0,49-1,10)$ & $0,86(0,58-1,28)$ \\
\hline 3 ou mais & 393 & $9,4(6,7-13,0)$ & $0,57(0,38-0,86)$ & $0,77(0,52-1,14)$ \\
\hline Hipertensão arterial & $0,000 * \star \star$ & & & \\
\hline Não & 1.005 & $15,7(12,6-19,3)$ & 1,00 & 1,00 \\
\hline $\operatorname{Sim}$ & 940 & $8,7(6,7-11,2)$ & $0,55(0,41-0,73)$ & $0,59(0,43-0,82)$ \\
\hline Diabetes mellitus & $0,099 * \star \star$ & & & \\
\hline Não & 1.654 & $12,9(10,6-15,6)$ & 1,00 & 1,00 \\
\hline Sim & 291 & $8,6(5,3-13,6)$ & $0,66(0,41-1,09)$ & $0,66(0,40-1,10)$ \\
\hline Acidente vascular encefálico & $0,059 * \star \star$ & & & \\
\hline Não & 1.861 & $11,9(9,8-14,2)$ & 1,00 & 1,00 \\
\hline Sim & 92 & $20,9(11,3-35,2)$ & $1,76(1,00-3,09)$ & $2,10(1,11-4,00)$ \\
\hline Depressão/Ansiedade & $0,027 \star \star \star$ & & & \\
\hline Não & 1.473 & $10,9(8,8-13,5)$ & 1,00 & 1,00 \\
\hline Sim & 476 & $16,2(11,9-21,6)$ & $1,48(1,05-2,08)$ & $2,36(1,55-3,59)$ \\
\hline Osteoporose & $0,004 * \star \star$ & & & \\
\hline Não & 1.660 & $13,3(11,0-16,0)$ & 1,00 & 1,00 \\
\hline Sim & 265 & $6,0(3,3-10,5)$ & $0,45(0,25-0,79)$ & $0,71(0,38-1,31)$ \\
\hline Doença crônica pulmão & $0,069 * \star \star$ & & & \\
\hline Não & 1.812 & $11,8(9,8-14,3)$ & 1,00 & 1,00 \\
\hline Sim & 142 & $17,8(11,4-26,8)$ & $1,50(0,97-2,32)$ & $1,65(1,08-2,51)$ \\
\hline Câncer & $0,274 * \star \star$ & & & \\
\hline Não & 1.890 & $12,1(10,0-14,5)$ & 1,00 & 1,00 \\
\hline $\operatorname{Sim}$ & 64 & $18,0(8,3-34,7)$ & $1,48(0,74-2,99)$ & $1,64(0,83-3,25)$ \\
\hline Doença do coração & $0,079 * \star \star$ & & & \\
\hline Não & 1.657 & $12,9(10,6-15,6)$ & 1,00 & 1,00 \\
\hline Sim & 297 & $8,8(5,7-13,4)$ & $0,68(0,44-1,05)$ & $0,90(0,59-1,40)$ \\
\hline Transtorno mental comum & $0,182 * \star \star$ & & & \\
\hline Ausente & 1.497 & $12,0(9,7-14,8)$ & 1,00 & 1,00 \\
\hline Presente & 378 & $15,1(11,1-20,3)$ & $1,26(0,90-1,77)$ & $1,88(1,34-2,65)$ \\
\hline
\end{tabular}

* Número de indivíduos na amostra não ponderada;

** Ajustado por idade, sexo e número de doenças;

$\star \star \star$ Valor de $\mathrm{p}$.

A exposição precoce ao fumo tem sido sistematicamente observada 37,39,40,41,42, assim como verificado em nosso estudo. Quase $43 \%$ dos idosos inquiridos haviam iniciado o comportamento tabágico antes dos 16 anos de idade, o que mostra a duração prolongada do consumo da droga e a provável maior dependência da nicotina 43 .

Além da idade de iniciação do tabagismo, o número de cigarros fumados e a avaliação do tempo de fumar o primeiro cigarro após acordar também são indicadores da intensidade da de- pendência nicotínica 38,43,44. O tempo de fumar o primeiro cigarro após acordar avalia quão rápido o fumante precisa de um cigarro pela manhã para elevar novamente os níveis séricos de nicotina para não experimentarem sintomas de abstinência 5,45 .

Quanto maior o consumo maior tende a ser a dependência pelo fato da nicotina ser uma droga psicoestimulante. A dopamina, a norepinefrina e outros hormônios psicoativos, liberados com o ato de fumar, dão ao tabagista uma sensação pra- 
Razões de prevalência (RP) brutas e ajustadas de fumantes em pessoas com 60 anos ou mais. Inquérito de Saúde no Estado de São Paulo (ISA-SP), 2001-2002.

\begin{tabular}{|c|c|c|}
\hline Variáveis e categorias & RP bruta (IC95\%) & RP ajustada * (IC95\%) \\
\hline \multicolumn{3}{|l|}{ Sexo } \\
\hline Masculino & 1,00 & 1,00 \\
\hline Feminino & $0,48(0,33-0,68)$ & $0,51(0,34-0,76)$ \\
\hline \multicolumn{3}{|l|}{ Faixa etária (anos) } \\
\hline $60-69$ & 1,00 & 1,00 \\
\hline $70-79$ & $0,59(0,40-0,89)$ & $0,57(0,38-0,85)$ \\
\hline 80 e mais & $0,39(0,21-0,72)$ & $0,34(0,16-0,73)$ \\
\hline \multicolumn{3}{|l|}{ Religião } \\
\hline Católica & 1,00 & 1,00 \\
\hline Evangélica & $0,23(0,09-0,57)$ & $0,29(0,12-0,73)$ \\
\hline Outras/Sem religião & $0,93(0,58-1,50)$ & $0,96(0,60-1,53)$ \\
\hline \multicolumn{3}{|l|}{ Renda per capita (salários mínimos) } \\
\hline$\leq 1$ & 1,00 & 1,00 \\
\hline$>1-2,5$ & $0,63(0,45-0,90)$ & $0,78(0,54-1,12)$ \\
\hline$>2,5-4$ & $0,61(0,37-0,98)$ & $0,84(0,50-1,40)$ \\
\hline$>4$ & $0,36(0,23-0,57)$ & $0,49(0,30-0,79)$ \\
\hline \multicolumn{3}{|l|}{ IMC } \\
\hline Normal $\left(22 \leq \mathrm{IMC} \leq 27 \mathrm{~kg} / \mathrm{m}^{2}\right)$ & 1,00 & 1,00 \\
\hline Baixo peso $\left(<22 \mathrm{~kg} / \mathrm{m}^{2}\right)$ & $2,53(1,84-3,47)$ & $1,91(1,42-2,57)$ \\
\hline Sobrepeso (> 27kg/m²) & $0,47(0,28-0,78)$ & $0,50(0,30-0,83)$ \\
\hline \multicolumn{3}{|l|}{ Atividade física de lazer } \\
\hline Não pratica & 1,00 & 1,00 \\
\hline Pratica & $0,40(0,26-0,60)$ & $0,40(0,25-0,64)$ \\
\hline \multicolumn{3}{|l|}{ Hipertensão arterial } \\
\hline Não & 1,00 & 1,00 \\
\hline Sim & $0,55(0,41-0,73)$ & $0,65(0,49-0,87)$ \\
\hline \multicolumn{3}{|l|}{ Depressão/Ansiedade } \\
\hline Não & 1,00 & 1,00 \\
\hline Sim & $1,48(1,05-2,08)$ & $1,76(1,27-2,45)$ \\
\hline
\end{tabular}

* Modelo de regressão múltipla de Poisson (variáveis que entraram no modelo para ajuste: sexo, faixa etária, cor/raça, religião, renda, atividade ocupacional, dependência de álcool, freqüência de ingestão de bebida alcoólica, índice de massa corporal, índice de qualidade da dieta, atividade física de lazer, número de doenças crônicas, hipertensão, diabetes, acidente vascular encefálico, depressão/ansiedade, osteoporose, doença crônica de pulmão, doença do coração, transtorno mental comum).

zerosa e tranquilizante. A nicotino-dependência, assim como a exposição continuada a outras drogas, leva à neuroadaptação e, consequentemente, à necessidade de aumento do consumo para a obtenção do mesmo efeito ${ }^{38}$. Em nosso trabalho, o consumo de 20 ou mais cigarros por dia foi encontrado em $45 \%$ dos fumantes idosos do sexo masculino e em $41,1 \%$ das idosas fumantes. Esse percentual masculino é inferior e o feminino superior aos observados em estudos de base populacional realizados no Canadá na década de 80, em que a proporção de idosos com
65 anos e mais que consumia mais de 20 cigarros por dia variou de $52 \%$ a $61 \%$ entre homens e de $30 \%$ a $38 \%$ em mulheres 46 . Na população de Bambuí, a proporção que consumia mais de 20 cigarros por dia foi bem inferior à encontrado em nosso estudo, sendo $9 \%$ dos homens e $5 \%$ das mulheres 16 .

A importância de influências culturais e do pertencimento a grupos é revelada pelas menores probabilidades de idosos evangélicos serem fumantes quando comparados aos católicos e aos de outras religiões ou sem religião. Menor preva- 
lência de fumantes entre os evangélicos também foi observada em estudos realizados em outros países 33,47,48,49 e no Brasil 39. Dalgalarrondo 49 relata que pessoas envolvidas em atividades religiosas e que frequentam cultos com assiduidade apresentam menores prevalências de uso, abuso ou dependência de substâncias psicotrópicas, e considera que, além do suporte social propiciado pelos grupos de devotos, as religiões tendem a incentivar alguns comportamentos considerados saudáveis. Ressalta ainda que as religiões protestantes, em especial, propõem princípios relacionados ao vestuário e ao comportamento sexual e de condenação do uso de álcool, tabaco e outras drogas. Em nosso trabalho, não foi possível avaliar o efeito da assiduidade em reuniões de cunho religioso pela inexistência destas informações no inquérito.

A maior prevalência de fumantes entre os idosos com menor renda também foi observada em outros estudos 11,16,43. Entretanto, o tabagismo não esteve associado de maneira significativa à escolaridade entre os idosos desta pesquisa. Esse resultado é semelhante ao encontrado em região metropolitana e em cidade de pequeno porte de Minas Gerais 11,16,29 e em três de cinco áreas metropolitanas da América Latina envolvidas em estudo multicêntrico, dentre elas São Paulo, em que não foram observadas diferenças significativas entre os diferentes níveis de escolaridade dos homens 37 . A associação negativa do fumo com a renda tem sido atribuída a algumas circunstâncias como o início mais precoce do tabagismo, a maior dependência e dificuldade de deixar o comportamento pela baixa motivação e pela falta de acesso a métodos adequados para a cessação do comportamento pelo segmento socialmente menos favorecido da população 11,16. Quanto à ausência de associação com a escolaridade, deve-se lembrar que a atual geração de idosos teve menor acesso à escola, de forma que a variável escolaridade tem menor poder que a renda de discriminar os estratos sociais neste segmento etário.

Idosos sedentários no lazer apresentaram maior probabilidade de serem fumantes. A coexistência de comportamentos não saudáveis em subgrupos da população já foi apontada em outros estudos, sendo observada a relação entre tabagismo, etilismo, sedentarismo e dieta inadequada $33,50,51,52$.

Uma observação importante desta pesquisa foi a constatação de prevalência elevada de tabagismo em idosos com doenças graves e mesmo tabaco relacionadas. Verificou-se $20,9 \%$ de fumantes nos idosos que haviam tido AVE, 18\% nos que tiveram câncer e $17,8 \%$ entre os que tinham uma doença crônica de pulmão. Especial atenção deve ser dada a esses grupos de idosos com doenças crônicas e que continuam a fumar - situação que contribui para ampliar ainda mais o risco de complicações e comorbidades - pois eles provavelmente apresentam dependência importante à nicotina e grande dificuldade para interromper o tabagismo.

Observou-se também associação entre comportamento de fumar e depressão/ansiedade referida, assim como relatado em estudos conduzidos em outras localidades 1,10,12,16,53,54,55,56. Sabe-se que a nicotina interfere no funcionamento neuroendócrino e, desta forma, pode influenciar o quadro psicopatológico. Provavelmente, as pessoas que relatam ansiedade e depressão sentem-se bem fumando porque a nicotina é ansiolítica e contribui para o alívio dos sintomas 54 . Cabe destacar que os sintomas de depressão podem ser um obstáculo na redução e interrupção do comportamento de fumar 10,12, embora esta observação seja controversa 57 .

Assim como em nossos achados, foram encontradas associações significativa e negativa de tabagismo e pressão alta em pesquisa com idosos do Rio Grande do Sul 33, em estudo canadense 46 e em mulheres da Região Metropolitana de Belo Horizonte ${ }^{11}$. A menor prevalência de tabagistas entre os hipertensos, comparada à dos não hipertensos, poderia indicar uma atuação positiva do serviço de saúde. O aumento da frequência aos serviços de saúde, induzido pela necessidade de controle da hipertensão, aumentaria a exposição dos pacientes a recomendações e intervenções educativas que incentivariam a cessação tabágica.

Observou-se prevalência muito elevada de fumantes entre os idosos com baixo peso $(42,9 \%)$ e mais baixa naqueles com sobrepeso, quando comparados aos de peso normal. Resultados consistentes da literatura registram que o peso corporal tende a ser mais baixo entre os fumantes 46,57 . Um dos mecanismos explicativos é que a dopamina e a serotonina liberadas pela nicotina atuam no controle e na regulação do apetite e da saciedade no nível do hipotálamo, ou ainda, que a nicotina aumenta a atividade adrenérgica, levando à maior dispêndio energético, maior consumo de calorias e, consequentemente, reduzindo o peso corporal 57.

O desenho deste estudo, que é de corte transversal, não permite estabelecer inferências causais e, portanto, a concluir, por exemplo, se o fumo desencadeou alterações químicas cerebrais levando à depressão/ansiedade ou se estes transtornos mentais colaboraram na manutenção do tabagismo entre os idosos. Isso também é válido ao considerar outras associações encontradas: enquanto o tabagismo é reconhecido como fator 
de risco para várias doenças, o diagnóstico da doença tenderia a levar à cessação do comportamento. Deve-se considerar também que os idosos fumantes deste estudo são os sobreviventes de uma coorte em que os indivíduos biológica e socialmente mais vulneráveis aos malefícios do tabagismo já faleceram 58 .

Outra limitação do trabalho diz respeito à obtenção de informações referidas. Dados sobre comportamentos socialmente indesejáveis são propensos a subestimativas. Particularmente sobre o tabagismo, o uso de questionário para avaliar o consumo de cigarros produz prevalências subestimadas quando comparadas às medidas realizadas com marcadores bioquímicos 59 . Estudos sugerem que o viés depende da população examinada, sendo as prevalências mais subestimadas entre aqueles em que fumar é visto como particularmente indesejável, como é o caso de mulheres grávidas e pessoas que apresentam doenças relacionadas ao tabaco 59,60. Apesar da possível subestimativa, nosso estudo detectou prevalências mais elevadas de tabagismo nos idosos com doenças tabaco relacionadas. Vários autores apontam ser válida essa forma de obter informação sobre o tabagismo 31,61, considerando o baixo custo do procedimento e a não exposição do entrevistado a constrangimentos ${ }^{59}$. As informações sobre peso e altura obtidas de pessoas idosas também são menos válidas para estimar o IMC do que em adultos, pois os idosos as afeririam com menor frequência e haveria tendência a subestimar o IMC 54. Neste estudo, utilizamos as categorias de IMC recomendadas para idosos, com pontos de corte que levam em consideração as mudanças na composição corporal decorrentes do envelhecimento 37 .

Este trabalho sinaliza que os idosos fumantes devem ser também alvo das políticas públicas de cessação do tabagismo, considerando as evidências existentes de que a descontinuação do comportamento traz benefícios em qualquer idade 1,12. O elevado percentual de fumantes, verificado nesta pesquisa, em idosos que já apresentam doenças crônicas, incluindo doenças respiratórias, neoplasia e AVE, apontam que os serviços de saúde devem prover atenção especial que garanta apoio, disponibilidade de tratamento e assistência em todo o processo de cessação do tabagismo para esses pacientes. Especialmente entre os fumantes com sintomas depressivos ou com predisposição para esta morbidade, é necessário prover acompanhamento já que terão mais dificuldade em cessar o tabagismo e uma maior propensão de recaídas caso haja interrupção 55,56.

A observação de prevalência quase três vezes maior de fumantes entre os idosos de menor renda familiar per capita (prevalência de 18,5\%) aponta o efeito de desigualdades sociais na saúde e a vulnerabilidade dos segmentos com piores condições socioeconômicas. Fica evidente, mais uma vez, a necessidade dos serviços de saúde organizarem-se e disponibilizarem tratamento, apoio e cuidados a esse segmento social.

O estudo, ao verificar a prevalência mais elevada do tabagismo nos idosos que praticam menos atividade física de lazer, apontou a convergência de comportamentos não saudáveis também em idosos, assinalando a necessidade de ações mais amplas que contemplem conjuntamente ou como parte das intervenções para a cessação do tabagismo, a realização de outras práticas saudáveis como exercícios durante o tempo livre, entre outras.

Os resultados do estudo apontam a necessidade de estratégias para cessação do tabagismo em idosos, com ações específicas para este grupo etário que se apresenta mais vulnerável a complicações e morte pela permanência do comportamento de fumar. 


\section{Resumo}

O objetivo deste trabalho foi determinar a prevalência do hábito de fumar e os fatores associados em idosos. Estudo transversal de base populacional, com amostragem em múltiplos estágios, que envolveu 1.954 idosos com 60 anos ou mais, residentes em quatro áreas do Estado de São Paulo, Brasil. A prevalência de fumantes foi de 12,2\%, sendo maior no sexo masculino, na faixa de 60 a 69 anos, nos estratos inferiores de renda, nos idosos com baixo peso corporal, nos que não praticavam atividade física de lazer, naqueles com depressão/ ansiedade e que referiram não ser hipertensos, e a prevalência foi menor entre os evangélicos. Prevalências elevadas de fumantes foram observadas em idosos com história de AVC, câncer e doença pulmonar crônica. Os resultados alertam para a necessidade de intervenções eficazes dos serviços de saúde para a cessação do tabagismo em idosos, visto que muitos deles, mesmo com doenças relacionadas ao tabaco, não conseguem deixar de fumar, especialmente junto ao estrato SUS dependente, pois a prevalência do tabagismo é maior nos segmentos socioeconômicos mais desfavorecidos.

Idoso; Tabagismo; Inquéritos de Morbidade; Promoção da Saúde

\section{Colaboradores}

M. P. A. Zaitune realizou a proposta do artigo, a revisão da literatura, a análise dos dados e a redação do artigo. M. B. A. Barros orientou a proposta do artigo, a análise dos dados e a redação do artigo. M. G. Lima colaborou na análise estatística e na redação do manuscrito. C. L. G. César, L. Carandina, M. Goldbaum e M. C. G. P. Alves contribuíram na revisão do artigo.

\section{Agradecimentos}

À Fundação de Amparo à Pesquisa do Estado de São Paulo (FAPESP) - Projeto de Políticas Públicas, processo $\mathrm{n}^{\circ} .88 / 14099$ e à Secretaria de Estado da Saúde de São Paulo pelo financiamento do trabalho de campo. À Secretaria de Vigilância em Saúde do Ministério da Saúde pelo suporte financeiro para a análise dos dados por intermédio do Centro Colaborador em Análise de Situação de Saúde da Faculdade de Ciências Médicas da Universidade Estadual de Campinas (FCM-UNICAMP). À Coordenação de Aperfeiçoamento de Pessoal de Nível Superior (CAPES) pela bolsa de doutorado recebida pela autora principal. M. B. A. Barros, C. L. G. César e M. Goldbaum recebem bolsa de produtividade do Conselho Nacional de Desenvolvimento Científico e Tecnológico (CNPq).

\section{Referências}

1. Doolan DM, Froelicher ES. Smoking cessation interventions and older adults. Prog Cardiovasc Nurs 2008; 23:119-27.

2. Kaczynski AT, Manske SR, Mannell RC, Grewal K. Smoking and physical activity: a systematic review. Am J Health Behav 2008; 32:93-110.

3. Oliveira AF, Valente JG, Leite IC. Aspectos da mortalidade atribuível ao tabaco: revisão sistemática. Rev Saúde Pública 2008; 42:335-45.

4. Instituto Nacional de Câncer. Programa Nacional de Controle do Tabagismo e Outros Fatores de Risco de Câncer: modelo lógico e avaliação. Rio de Janeiro: Instituto Nacional de Câncer; 2003.

5. Meneses-Gaya IC, Zuardi AW, Loureiro SR, Crippa JAS. As propriedades psicométricas do Teste de Fagerström para Dependência de Nicotina. J Bras Pneumol 2009; 35:73-82.

6. Organização Pan-Americana da Saúde. Saúde nas Américas: 2007. Washington DC: Organização PanAmericana da Saúde; 2007.
7. World Health Organization. Report on the Global Tobacco Epidemic 2008: the MPOWER package. Geneva: World Health Organization; 2008.

8. Anand P, Kunnumakkara AB, Sundaram C, Harikumar KB, Tharakan ST, Lai OS, et al. Cancer is a preventable disease that requires major lifestyle changes. Pharm Res 2008; 25:2097-116.

9. Centers for Disease Control and Prevention. Statespecific smoking-attributable mortality and years of potential life lost: United States, 2000-2004. MMWR Morb Mortal Wkly Rep 2009; 58:29-33.

10. Kenney BA, Holahan CJ, Holahan CK, Brennan PL, Schutte KK, Moos RH. Depressive symptoms, drinking problems, and smoking cessation in older smokers. Addict Behav 2009; 34:548-53.

11. Peixoto SV, Firmo JOA, Lima-Costa MF. Condições de saúde e tabagismo entre idosos residentes em duas comunidades brasileiras (Projetos Bambuí e Belo Horizonte). Cad Saúde Pública 2006; 22: 1925-34. 
12. Sachs-Ericsson N, Schmidt NB, Zvolensky MJ, Mitchell M, Collins N, Blazer DG. Smoking cessation behavior in older adults by race and gender: the role of health problems and psychological distress. Nicotine Tob Res 2009; 11:433-43.

13. Galán I, Rodriguez-Artalejo F, Tobías A, Díez-Gañán L, Gandarilhas A, Zorrilla B. Agregación de factores de riesgo ligados al comportamiento y su relación con la salud subjetiva. Gac Sanit 2005; 19:1-9.

14. Gómez-Gutiérrez LF, Lucumí-Cuesta DI, GirónVargas SL, Espinosa-García G. Conglomeración de factores de riesgo de comportamiento asociados a enfermedades crónicas en adultos jóvenes de dos localidades de Bogotá, Colombia: importancia de las diferencias de género. Rev Esp Salud Pública 2004; 78:493-504.

15. Berto SJP, Carvalhaes MABL, Moura EC. Tabagismo associado a outros fatores comportamentais de risco de doenças e agravos crônicos não transmissíveis. Cad Saúde Pública 2010; 26:1573-82.

16. Peixoto SV, Firmo JOA, Lima-Costa MF. Factors associated to smoking habit among older adults (The Bambuí Health and Aging Study). Rev Saúde Pública 2005; 39:746-53.

17. Carvalho JAM, Garcia RA. O envelhecimento da população brasileira: um enfoque demográfico. Cad Saúde Pública 2003; 19:725-33.

18. Veras R. Envelhecimento populacional contemporâneo: demandas, desafios e inovações. Rev Saúde Pública 2009; 43:548-54.

19. Araújo AJ, Menezes AMB, Dórea AJPS, Torres BS, Viegas CAA, Silva CAR, et al. Diretrizes para cessação do tabagismo. J Bras Pneumol 2004; 30 Suppl 2:S2-76.

20. César CLG, Carandina L, Alves MCP, Barros MBA, Goldbaum M, organizadores. Saúde e condição de vida em São Paulo: Inquérito Multicêntrico de Saúde no Estado de São Paulo - ISA/SP. São Paulo: Faculdade de Saúde Pública, Universidade de São Paulo; 2005.

21. Alves MCGP. Plano de amostragem. In: César CLG, Carandina L, Alves MCP, Barros MBA, Goldbaum M, organizadores. Saúde e condição de vida em São Paulo: Inquérito Multicêntrico de Saúde no Estado de São Paulo - ISA/SP. São Paulo: Faculdade de Saúde Pública, Universidade de São Paulo; 2005. p. 47-62.

22. Fisberg RM, Slater B, Barros RR, Lima FD, César CLG, Carandina L, et al. Índice de qualidade da dieta: avaliação da adaptação e aplicabilidade. Rev Nutr 2004; 17:301-8.

23. Bisson J, Nadeau L, Demers A. The validity of the CAGE scale to screen for heavy drinking and drinking problem in a general population survey. Addiction 1999; 94:715-22.

24. Matsudo S, Araújo T, Matsudo V, Andrade D, Andrade E, Oliveira LC, et al. Questionário Internacional de Atividade Física (IPAQ): estudo de validade e reprodutibilidade no Brasil. Rev Bras Ativ Fís Saúde 2001; 6:5-18.

25. World Health Organization. A user's guide to the Self Reporting Questionnaire. Geneva: World Health Organization; 1994.

26. Cervi A, Franceschni SCC, Priore SE. Análise crítica do uso do índice de massa corporal para idosos. Rev Nutr 2005; 18:765-75.
27. Barros AJ, Hirakata VN. Alternatives for logistic regression in cross-sectional studies: an empirical comparison of models that directly estimate the prevalence ratio. BMC Med Res Methodol 2003; 3:21.

28. Paskulin LMG, Vianna LAC. Perfil sociodemográfico e condições de saúde auto-referidas de idosos de Porto Alegre. Rev Saúde Pública 2007; 41: 757-68.

29. Lima-Costa MF. A escolaridade afeta, igualmente, comportamentos prejudiciais à saúde de idosos e adultos mais jovens? Inquérito de Saúde da Região Metropolitana de Belo Horizonte, Minas Gerais, Brasil. Epidemiol Serv Saúde 2004; 13:201-8.

30. Instituto Brasileiro de Geografia e Estatística. Pesquisa Nacional do Tabagismo - Petab. Rio de Janeiro: Instituto Brasileiro de Geografia e Estatística; 2009. (Suplemento Saúde da Pesquisa Nacional por Amostra de Domicílios, 2008).

31. Pereira JC, Barreto SM, Passos VMA. O perfil de saúde cardiovascular dos idosos brasileiros precisa melhorar: estudo de base populacional. Arq Bras Cardiol 2008; 91:1-10.

32. Szwarcwald CL, Viacava F, Vasconcellos MTL, Leal MC, Azevedo LO, Queiroz RSB, et al. Pesquisa Mundial de Saúde 2003: o Brasil em números. RADIS Comunicação em Saúde 2004; 23:14-33.

33. Marinho V, Blay SL, Andreoli SB, Gastal F. A prevalence study of current tobacco smoking in later life community and its association with sociodemographic factors, physical health and mental health status. Soc Psychiatry Psychiatr Epidemiol 2008; 43:490-7.

34. Carvalhaes MABL, Moura EC, Monteiro CA. Prevalência de fatores de risco para doenças crônicas: inquérito populacional mediante entrevistas telefônicas em Botucatu, São Paulo, 2004. Rev Bras Epidemiol 2008; 11:14-23.

35. Centers for Disease Control and Prevention. Cigarette smoking among adults: United States, 2007. MMWR Morb Mortal Wkly Rep 2008; 57:1221-6.

36. Moura EC, Silva SA, Malta DC, Morais Neto OL. Fatores de risco e proteção para doenças crônicas: vigilância por meio de inquérito telefônico, VIGITEL, Brasil, 2007. Cad Saúde Pública 2011; 27:486-96.

37. Menezes AM, Lopez M, Hallal PC, Muino A, PerezPadilla R, Jardim J, et al. Prevalence of smoking and incidence of initiation in the Latin American adult population: the PLATINO study. BMC Public Health 2009; 9:151.

38. Rosemberg J, Rosemberg AMA, Moraes MA. Nicotina: droga universal. São Paulo: Centro de Vigilância Epidemiologia, Secretaria de Estado da Saúde de São Paulo; 2003.

39. Souza AAF, Barros MBA. Tabagismo. In: Barros MBA, César CLG, Carandina L, Goldbaum M, organizadores. As dimensões da saúde: inquérito populacional em Campinas. São Paulo: Editora Hucitec; 2008. p. 80-99.

40. Drum ML, Shiovitz-Ezra S, Gaumer E, Lindau ST. Assessment of smoking behaviors and alcohol use in the national social life, health, and aging project. J Gerontol B Psychol Sci Soc Sci 2009; 64 Suppl 1:1119-30. 
41. Borges MTT, Barbosa RHS. As marcas de gênero no fumar feminino: uma aproximação sociológica do tabagismo em mulheres. Ciênc Saúde Coletiva 2009;14:1129-39.

42. Santos SR, Gonçalves MS, Leitão FF, Sergio S, Jardim JR. Perfil dos fumantes que procuram um centro de cessação de tabagismo. J Bras Pneumol 2008; 34:695-701.

43. Marcopito LF, Coutinho AP, Valencich DMO, Moraes MA, Brumini R, Ribeiro SA. Exposição ao tabagismo e atitudes: comparação entre inquéritos realizados na população adulta do Município de São Paulo em 1987 e 2002. Arq Bras Cardiol 2007; 89:333-40.

44. Halty LS, Hüttner MD, Oliveira Netto I, Fenker T, Pasqualini T, Lempek B, et al. Pesquisa sobre tabagismo entre médicos de Rio Grande, RS: prevalência e perfil do fumante. J Pneumol 2002; 28:77-83.

45. Halty LS, Hüttner MD, Oliveira Netto IC, Santos VA, Martins G. Análise da utilização do Questionário de Tolerância de Fagerström (QTF) como instrumento de medida da dependência nicotínica. J Pneumol 2002; 28:180-6.

46. Maxwell CJ, Hirdes JP. The prevalence of smoking and implications for quality of life among the community-based elderly. Am J Prev Med 1993; 9:338-45.

47. Gilmore AB, McKee M, Telishevska M, Rose R. Epidemiology of smoking in Ukraine. Prev Med 2000; 33:453-61.

48. Williams CD, Lewis-Jack O, Johnson K, AdamsCampbell L. Environmental influences, employment status, and religious activity predict current cigarette smoking in the elderly. Addict Behav 2001; 26:297-301

49. Dalgalarrondo P. Relações entre duas dimensões da vida: saúde mental e religião. Rev Bras Psiquiatr 2006; 28:177-8.

50. Crespo CJ, Smit E, Carter-Pokras O, Andersen R. Acculturation and leisure-time physical inactivity in Mexican Americans adults: results from NHANES III, 1988-1994. Am J Public Health 2001; 91:1254-7.

51. Paavola M, Vartiainen E, Haukkala A. Smoking, alcohol use, and physical activity: a 13-year longitudinal study ranging from adolescence into adulthood. J Adolesc Health 2004; 35:238-44.
52. Gazalle FK, Lima MS, Tavares BF, Hallal PC. Sintomas depressivos e fatores associados em população idosa no Sul do Brasil. Rev Saúde Pública 2004; 38:365-71.

53. Varo JJ, Martínez-González MA, Irala-Estévez J, Kearney J, Gibney M, Martínez JA. Distribuion and determinants of sedentary lifestyles in the European Union. Int J Epidemiol 2003; 32:138-46.

54. Rondina RC, Gorayeb R, Botelho C. Características psicológicas associadas ao comportamento de fumar tabaco. J Bras Pneumol 2007; 33:592-601.

55. Khaled S, Bulloch A, Exner D, Patten S. Cigarette smoking, stages of change, and major depression in the Canadian population. Can J Psychiatry 2009; 54:204-8.

56. Chatkin R, Chatkin JM. Tabagismo e variação ponderal: a fisiopatologia e genética podem explicar esta associação? J Bras Pneumol 2007; 33:712-9.

57. Kinnunen T, Haukkala A, Korhonen T, Quiles ZN, Spiro 3rd A, Garvey AJ. Depression and smoking across 25 years of the Normative Aging Study. Int J Psychiatry Med 2006; 36:413-26.

58. Lima-Costa MF, Barreto SM. Tipos de estudos epidemiológicos: conceitos básicos e aplicações na área do envelhecimento. Epidemiol Serv Saúde 2003; 12:189-201.

59. Gorber SC, Schofield-Hurwitz S, Hardt J, Levasseur G, Tremblay M. The accuracy of self-reported smoking: A systematic review of the relationship between self-reported and cotinine-assessed smoking status. Nicotine Tob Res 2009; 11:12-24.

60. Attebring MF, Herlitz J, Berndt AK, Karlsson T, Hjalmarson A. Are patients truthful about their smoking habits? A validation of self-report about smoking cessation with biochemical markers of smoking activity amongst patients with ischaemic heart disease. J Intern Med 2001; 249:145-51.

61. Kahende JW, Adhikari B, Maurice E, Rock V, Malarcher A. Disparities in health care utilization by smoking status - NHANES 1999-2004. Int J Environ Res Public Health 2009; 6:1095-106.

Recebido em 20/Jul/2010

Versão final reapresentada em 01/Jul/2011

Aprovado em 21/Nov/2011 